\title{
Moving up and down in the generic multiverse
}

\author{
Joel David Hamkins ${ }^{1,2}$, Benedikt Löwe ${ }^{3,4 \star}$ \\ 1 Mathematics Program, The Graduate Center of The City University of New York, \\ 365 Fifth Avenue, New York, NY 10016, U.S.A.; jhamkins@gc.cuny.edu \\ 2 Department of Mathematics, The College of Staten Island of CUNY, Staten Island, \\ NY 10314, U.S.A., \\ 3 Institute for Logic, Language and Computation, Universiteit van Amsterdam, \\ Postbus 94242, 1090 GE Amsterdam, The Netherlands; b.loewe@uva.nl \\ 4 Department Mathematik, Universität Hamburg, Bundesstrasse 55, 20146 Hamburg, \\ Germany
}

\begin{abstract}
We investigate the modal logic of the generic multiverse which is a bimodal logic with operators corresponding to the relations "is a forcing extension of" and "is a ground model of". The fragment of the first relation is the modal logic of forcing and was studied by the authors in earlier work. The fragment of the second relation is the modal logic of grounds and will be studied here for the first time. In addition, we discuss which combinations of modal logics are possible for the two fragments.
\end{abstract}

\section{Introduction}

\subsection{The generic multiverse}

Recently, the generic multiverse has become a concept of great interest to set theorists and philosophers of mathematics alike:

The generic multiverse is generated from each universe of the collection by closing under generic extensions (enlargements) and under generic refinements (inner models of a universe which the given universe is a generic extension of). (W. Hugh Woodin, [21])

If you fix a universe of set theory $V$ and iteratively build all set forcing extensions and ground models - and throughout this article unless stated specifically otherwise we shall mean always set forcing when discussing forcing-you naturally produce a Kripke structure $\operatorname{GM}(V)$ consisting of these worlds together with the accessibility relation $M \sqsubseteq N$ (" $N$ is a forcing extension of $M$ ") and

\footnotetext{
* The research of the first author has been supported in part by NSF grant DMS0800762, PSC-CUNY grant 64732-00-42 and Simons Foundation grant 209252. The second author would like to thank the CUNY Graduate Center in New York for their hospitality during his sabbatical in the fall of 2009. In addition, both authors acknowledge the generous support provided to them as visiting fellows in Spring 2012 at the Isaac Newton Institute for Mathematical Sciences in Cambridge, U.K.
} 
its converse relation $N \sqsupseteq M$ (" $M$ is a ground of $N$ "). Hugh Woodin has investigated multiverse truth, that is, truth in all models of the generic multiverse, in connection with his programme to solve the alethic status of the continuum hypothesis. The first author has proposed a philosophy of mathematics based on a broader multiverse perspective, where we have many different legitimate concepts of set (not merely those arising by set forcing), and these are instantiated in their corresponding set-theoretic universes, which relate in diverse ways as forcing extensions, large cardinal ultrapowers, definable inner models and so on 9. Although the generic multiverse of a model of set theory is merely a local neighborhood within the broader multiverse, nevertheless we take it that our project in this article, to study the forcing modalities of the generic multiverse, surely engages with the multiverse perspective.

Due to the fact that the relations $\sqsubseteq$ and $\sqsupseteq$ are converses of each other, the bimodal logic of the Kripke structure $(\operatorname{GM}(V), \sqsubseteq, \sqsupseteq)$ is similar to temporal logics where the modality $\mathbf{F}$ ("there is a time in the future") is converse to the modality $\mathbf{P}$ ("there is a time in the past") 20. It is our overall aim to investigate this bimodal logic of the general multiverse and find out which validities hold in general (provably in ZFC) or in the multiverse generated from specific universes.

\subsection{The modal logic of forcing and related work}

In our previous paper [11, we introduced the modal logic of forcing and proved that the ZFC-provably valid principles of forcing were exactly those in the modal theory known as S4.2. The modal logic of forcing corresponds to the monomodal fragment of the modal logic discussed in $\S 1.1$ that only uses the relation $\sqsubseteq$; or to the part of the generic multiverse that is generated only by the operation of taking forcing extensions and not ground models.

In [11, we not only consider the ZFC-provable modal logic of forcing, but also the modal logic of forcing of particular universes $V$. We show that this modal logic always lies between S4.2 and S5 and that the two extreme values are realized (for more details, cf. $\oint$ [1.4). Various other aspects of the modal logic of forcing are considered in 15$] 12[3 / 4] 16[19] 2|1| 10$. The paper [8 presented at ICLA 2009 gives an overview of the status of research and creates a connection between the modal logic of forcing and "set-theoretic geology", i.e., going down from a universe to its ground models. This connection is further developed in this paper.

\subsection{The results of this paper}

As stated above, our overall aim is to understand the bimodal logic of the generic multiverse. However, we know rather little about the general status of the bimodal logic (cf. Footnote 17). Instead of the bimodal theory, we consider the two monomodal fragments (the modal logic of forcing and the modal logic of grounds) and their possible combinations.

The main result of this paper is Theorem [6, constructing a model of set theory whose modal logic of grounds is S4.2. This theorem is the downward analogue 
of the main result in [11, where we showed that the modal logic of forcing over $\mathbf{L}$ is S4.2. Based on the proof idea of the main result, we then make a foray into the bimodal world, by considering which combinations are possible for the two monomodal fragments; i.e., for which pairs $\left(\Lambda, \Lambda^{*}\right)$ can we find a model $V$ such that $\mathrm{ML}(\square, V)=\Lambda$ and $\mathrm{ML}(\nabla, V)=\Lambda^{*}$. We consider all possible combinations with $\Lambda, \Lambda^{*} \in\{\mathrm{S} 4.2, \mathrm{~S} 5\}$.

The paper is organized as follows: in $\$ 1.4$, we give the necessary definitions in order to discuss the basic properties of the modal logic of grounds (and how it differs from the modal logic of forcing) in $\S 2$. In $\S$, we finally consider the various combinations of upward and downward modal logics. The paper is not self-contained and uses ideas and concepts from the papers [11|18; the proofs of our theorems are sketches and will be presented in more detail in the journal version of the paper.

\subsection{Definitions}

In the following, we denote by $\mathrm{P}$ a countable set of propositional letters; for modal operators $\Delta$ and $\nabla, \mathcal{L}_{\bowtie}$ and $\mathcal{L}_{\square, \nabla}$ are the monomodal and bimodal propositional languages with the appropriate operators. We assume that the reader is familiar with the standard axioms and systems of monomodal logic, in particular, .2, S4, S4.2, and S5 (if not, there is a summary in [11, §1]).

By $\mathcal{L}_{\epsilon}$, we denote the first-order language of set theory with its set of sentences $\operatorname{Sent}\left(\mathcal{L}_{\in}\right)$. Any function $I: \mathrm{P} \rightarrow \operatorname{Sent}\left(\mathcal{L}_{\epsilon}\right)$ is called an interpretation. An interpretation $I$ generates a valuation of any $\operatorname{Kripke}$ structure $(F, R)$ in which the worlds consist of models $M$ of set theory and $R$ is any relation between them: via

$$
I^{*}(M):=\{p ; M \models I(p)\},
$$

$\left(F, R, I^{*}\right)$ becomes a Kripke model (similarly, for the bimodal language if we have two relations on $F$ ).

Note that in our special case (when $R=\sqsubseteq$ ), the validity of a modal formula at a world of the Kripke model is not just a meta-theoretic property of the Kripke model, but can be expressed in the language of set theory: e.g., $\Delta \varphi$ is interpreted by "for all generic extensions, $\varphi$ holds" which by the Forcing Theorem [13, Theorem 14.6] is equivalent to "for all Boolean algebras $\mathbb{B}$, we have that $\llbracket \varphi \rrbracket_{\mathbb{B}}=1$ ". Similarly, if $R=\sqsupseteq$, we can use a theorem of Laver's (cf. [14]) that the ground model is definable with parameters in the forcing extension, in order to see that "for all ground models, $\varphi$ holds" is expressible in the language of set theory (this observation is due to Reitz, cf. [5. Theorem 8]).

These observations are closely related to Woodin's result about multiverse truth:

Theorem 1 (Woodin, 2009) There is a recursive transformation $\varphi \mapsto \varphi^{*}$ such that $M \models \varphi^{*}$ is equivalent to the statement "for every model $N$ in the generic multiverse generated by $M, \varphi$ is true". 
The fact that the modalities are expressible in the language of set theory allows us to move from interpretations to translations: We call a function $H$ : $\mathcal{L}_{\square, \nabla} \rightarrow \operatorname{Sent}\left(\mathcal{L}_{\in}\right)$ a translation if

- $H(\varphi \wedge \psi)=H(\varphi) \wedge H(\psi)$,

$-H(\neg \varphi)=\neg H(\varphi)$,

- $H(\Delta \varphi)$ is the sentence stating "for all forcing extensions $M$, we have $M \models$ $H(\varphi)$ ", and

- $H(\nabla \varphi)$ is the sentence stating "for all grounds $M$, we have $M \models H(\varphi)$ ".

Now, we can define the modal logic of the multiverse and two of its fragments: fixing a universe $V$, we call

$$
\operatorname{ML}(\square, \nabla, V):=\left\{\varphi \in \mathcal{L}_{\square, \nabla} ; \text { for all translations } H, V \models H(\varphi)\right\}
$$

the modal logic of the generic multiverse generated by $V$. Similarly,

$$
\begin{aligned}
& \mathrm{ML}(\square, V):=\left\{\varphi \in \mathcal{L}_{\square} ; \quad \text { for all translations } H, V \models H(\varphi)\right\}, \text { and } \\
& \operatorname{ML}(\nabla, V):=\left\{\varphi \in \mathcal{L}_{\square} ; \text { for all translations } H, V \models H(\varphi)\right\}
\end{aligned}
$$

are the modal logic of forcing and the modal logic of grounds at $V$, respectively. Metaphorically, we think of forcing extensions going upwards and thus the relation being a ground model going downwards. We therefore use the words "upward" and "downward" to indicate which modalities we are talking about: e.g., if we say that a model $V$ satisfies upward $\mathrm{S} 4.2$, we mean that $\mathrm{ML}(\Delta, V)=\mathrm{S} 4.2$; similarly, we talk of "upward buttons" and "downward buttons" (see below).

As mentioned, in [11, Theorem 21], we proved that for any universe $V$, we get that

$$
\mathrm{S} 4.2 \subseteq \mathrm{ML}(\triangle, V) \subseteq \mathrm{S} 5,
$$

and that the two extreme values are obtained. This immediately implies that the ZFC-provable modal logic of forcing

$$
\left\{\varphi \in \mathcal{L}_{\square} ; \text { for all translations } H, \mathrm{ZFC} \vdash H(\varphi)\right\}
$$

is exactly S4.2. Two facts about forcing are crucial for this result: the first is that the axiom .2 is always a validity for the modal logic of forcing over any universe $V$ (cf. [15] and [10, Theorem 7] for the theoretical background), providing the lower bound; the second is the existence of independent switches over each model of set theory [11, Theorem 17]. We shall see that the situation is quite different for the modal logic of grounds. Note that it is not known whether the modal logic of forcing can obtain any other value than S4.2 or S5 [11, Question 19].

In order to show upper bounds for a modal logic, we used certain control statements called buttons and switches: a switch is a statement $\varphi$ such that $\varphi$ and $\neg \varphi$ are both necessarily possible. A button is a statement $\varphi$ that is necessarily possibly necessary. These controls are independent if they can be operated independently, without affecting the status of the others (cf. [11, p. 1789] or [10, $\S 4]$ for more detail). We shall use the abstract results that produce upper 
bounds from the existence of control statements in the proof sketches in this paper. For this we call a function $\sigma: \mathrm{P} \rightarrow \mathcal{L}_{\square}$ a substitution (this is the purely modal version of our notion of interpretation above); every substitution induces a function $\hat{\sigma}$ on the entire set of modal formulas. If $(F, R, v, w)$ is a pointed Kripke model, we let $\operatorname{ML}(F, R, v, w):=\left\{\varphi \in \mathcal{L}_{\square}\right.$; for all substitutions $\sigma$, we have that $F, R, v, w \models \hat{\sigma}(\varphi)\}$.

Theorem 2 If a pointed reflexive and transitive Kripke model $(F, R, v, w)$ has arbitrarily large finite independent families of buttons and switches, then

$$
\operatorname{ML}(F, R, v, w) \subseteq \mathrm{S} 4.2
$$

[10, Theorem 13].

Theorem 3 If a pointed reflexive and transitive Kripke model $(F, R, v, w)$ has arbitrarily large finite independent families of switches, then

$$
\operatorname{ML}(F, R, v, w) \subseteq \mathrm{S} 5
$$

[10, Theorem 10].

\section{Basic Results about the modal logic of grounds.}

It is easy to see that every S4 assertion is downward valid (since a ground of a ground is a ground), but things are not as easy with the axiom .2. This axiom would be valid if the answer to the following question is "Yes":

Question 4 Let $V$ be a model of set theory, and $M$ and $N$ two grounds of $V$, i.e., $V=M[G]=N[H]$ for some generic filters $G$ and $H$. Is there some model $K$ which is a ground of both $M$ and $N$, i.e., there are $K$-generic filters $G^{*}$ and $H^{*}$ such that $K\left[G^{*}\right]=M$ and $K\left[H^{*}\right]=N$ ? In other words, is $\sqsupseteq$ directed among the grounds of $V$ ?

However, we do not know the answer to this question. We shall say that a universe $V$ in whose generic multiverse the answer to Question 4 is "Yes" satisfies the axiom of downward directedness of grounds (DDG). In all universes for which we can determine the truth value of DDG, it is true.

The upper bound for the modal logic of forcing was S5, but the situation for the modal logic of grounds is different. We denote by PL (for "propositional logic") the modal logic satisfying $\square p \leftrightarrow \diamond p \leftrightarrow p$, i.e., the modal logic of a single reflexive point. By GA, we denote the ground axiom of [18 stating that the universe is not a non-trivial forcing extension of an inner model 5 Clearly, the constructible universe satisfies GA.

Observation 5 If $V \models \mathrm{GA}$, then $\mathrm{ML}(\square, V)=\mathrm{PL}$.

\footnotetext{
${ }^{5}$ The ground axiom is jointly due to Reitz and the first author; cf. [7]17.
} 


\section{$3 \quad$ S4.2 as the modal logic of grounds}

The following theorem provides us with a model in which we can determine the modal logic of grounds. Its proof will serve as the underlying idea for the results

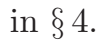

Theorem 6 If ZFC is consistent, then there is a model of ZFC whose ground valid assertions are exactly those in the modal theory S4.2.

Proof sketch. The idea of this proof is to use the bottomless model of [18: Let $\operatorname{Reg}^{\mathbf{L}}$ denote the class of regular $\mathbf{L}$-cardinals. Force over $\mathbf{L}$ with

$$
\mathbb{P}=\prod_{\gamma \in \operatorname{Reg}^{\mathbf{L}}} \operatorname{Add}(\gamma, 1),
$$

where we use Easton support and we use $\operatorname{Add}(\gamma, 1)$ as defined in L. Let $V=$ $\mathbf{L}[G]$, where $G$ is $\mathbf{L}$-generic for this forcing.

First, following Reitz, we argue that every ground model of $V$ contains a tail $\mathbf{L}\left[G^{\alpha}\right]$, where $G^{\alpha}=G \uparrow \mathbb{P}^{\alpha}$, and $\mathbb{P}^{\alpha}=\mathbb{P} \uparrow[\alpha, \infty)$. That is, $\mathbb{P}^{\alpha}$ is the tail forcing, using only the factors from $\alpha$ onward. Suppose that $W$ is a ground of $V$, so that $W[H]=V=\mathbf{L}[G]$. If the filter $G^{\alpha}$ is not in $W$, then it has a name there, and the Boolean value of the statement that this name is decided in certain ways compatible with the actual values of $G^{\alpha}$ will be a strictly descending sequence of Boolean values in the $W$-forcing, which violates the chain condition of that forcing (when $\alpha$ is much larger than that forcing). So, for large $\alpha, G^{\alpha}$ is an element of $W$.

In 18, Reitz used this to show that $V$ has no bedrock, and we use the same argument to show that for any two grounds $M$ and $N$, we find $\alpha$ and $\beta$ such that $\mathbf{L}\left[G^{\alpha}\right]$ is a ground of $M$ and $\mathbf{L}\left[G^{\beta}\right]$ is a ground of $N$. Then if $\mu:=\max \{\alpha, \beta\}$, $\mathbf{L}\left[G^{\mu}\right]$ is a ground of both $M$ and $N$. This proves that DDG holds in $V$, and thus .2.

We now show that there are no additional modal validities by using Theorem 2. Divide the regular cardinals above $\aleph_{\omega}$ into $\omega$ many disjoint classes $\Gamma_{n}$, each containing unboundedly many cardinals. Enumerate each class $\Gamma_{n}=\left\{\gamma_{\alpha}^{n} ; \alpha<\right.$ Ord $\}$ in order. Let $s_{n}$ be the statement "the least $\alpha$ such that there exists an L-generic subset of $\gamma_{\alpha}^{n}$ is even." These statements are all true in $V$, since the corresponding $\alpha$ is 0 in every case, as the forcing $G$ explicitly adds an $\mathbf{L}$-generic subset of every $\gamma_{\alpha}^{n}$, including $\alpha=0$. In any ground model $W$ of $V$, we can go to a deeper ground which is a tail extension, and then selectively remove additional factors of $G$ on indices in each $\Gamma_{n}$ so as to realize any desired configuration of the switches in $L$. So the $s_{n}$ 's are independent switches. Now let $b_{n}$ be the statement: "there is no $\mathbf{L}$-generic subset of $\aleph_{n}^{\mathbf{L}}$ ". This statement is false in $V$, but true in any ground model of $V$ omitting the factor at $\aleph_{n}^{\mathbf{L}}$. Furthermore, once true, the statement remains true in any deeper ground. Thus, each $b_{n}$ is a button. Finally, all these buttons and switches are independent, because each is controlled by re- 
moving disjoint factors of $G 6$ Thus, Theorem 2 yields that $\operatorname{ML}(\nabla, V)=\mathrm{S} 4.2$.

It follows that the ZFC-provably valid principles of the modal of logic of grounds is a theory containing S4 and contained within S4.2. If DDG is a theorem of ZFC, the ZFC-provable modal logic of grounds is exactly S4.2.

\section{Combinations}

So far, we have looked at the modal logic of forcing and the modal logic of grounds in isolation, but ultimately, we are interested in determining the entire bimodal logic of the multiverse. Currently, we know almost nothing about the validity of mixed bimodal formulas beyond those validities that follow from the fact that the modal operators $\Delta$ and $\nabla$ are defined by converse relations 7 However, we can say something about possible combinations of modal logics of forcing with modal logics of grounds (in the case of Theorems 8 and 9 under mild large cardinal assumptions).

Theorem 7 If ZFC is consistent, then there is a model of ZFC whose modal logic of forcing and modal logic of grounds are both S4.2.

Proof sketch. In fact, this is the model $V$ constructed in the proof of Theorem [6. since S4.2 is a general lower bound for the modal logic of forcing, we only have to show that independent upward buttons and switches exist. We have such a family for $\mathbf{L} 8$ and by observing that the forcing to add $G$ was cardinalpreserving and the GCH holds, we can use the buttons proposed by Rittberg [19] or Friedman, Fuchino and Sakai [2] or, alternatively, our stationary buttons from [11, Theorem 29] (provided that we start the other forcing above $\omega_{1}$ ). The switches are GCH at $\aleph_{\omega+n}$.

Theorem 8 If ZFC+ "there is an inaccessible cardinal $\delta$ in $\mathbf{L}$ such that $\mathbf{L}_{\delta}<\mathbf{L}$ " is consistent, then there is a model of set theory whose modal logic of forcing is S4.2 and whose modal logic of grounds is S5.

Proof sketch. This proof is a combination of the construction in Theorem 6 and an idea from [6], Theorem 5]. We start with an inaccessible cardinal $\delta$ in $\mathbf{L}$ such that $\mathbf{L}_{\delta}<\mathbf{L}$ and force as in the proof of Theorem 6 with the Easton support product $\mathbb{P}:=\prod_{\gamma \in \operatorname{Reg} \mathbf{L}} \operatorname{Add}(\gamma, 1)$ to obtain $\mathbf{L}[G]$. Since $\delta$ was inaccessible in $\mathbf{L}$, $\mathbb{P}_{\delta}$ is just $\mathbb{P}$ as defined in $\mathbf{L}_{\delta}$ and we took a direct limit at $\delta$; thus, we still

${ }^{6}$ Note that $\mathbf{L}$-generic Cohen subsets of different regular cardinals in $\mathbf{L}$ are necessarily mutually generic.

${ }^{7}$ E.g., we know that $p \rightarrow \Delta \neg \nabla \neg p$ and $p \rightarrow \nabla \neg \Delta \neg p$ hold.

${ }^{8}$ Note that the buttons provided in the proof of [11, Lemma 6.1] are problematic since we do not know how to prove their independence, but there are other independent buttons in that paper. Cf. the discussion at the end of [10, $\S 4$ ]. 
have $\mathbf{L}_{\delta}\left[G_{\delta}\right]<\mathbf{L}[G]$. We claim that $V:=\mathbf{L}\left[G^{\delta}\right]$ satisfies the conclusion of the theorem.

Upward S4.2 follows as in the proof of Theorem 7 . Let us show that all downward buttons are pushed (this will establish S5 as a lower bound for the modal logic of grounds): If there is a ground pushing a downward button, then this fact is expressible, and so there must be a ground of $\mathbf{L}_{\delta}\left[G_{\delta}\right]$ pushing that button, and so this same forcing works with $\mathbf{L}[G]$. So there is a small forcing pushing that button. And this small forcing ground will contain $\mathbf{L}\left[G^{\delta}\right]$, so it is already pushed in $\mathbf{L}\left[G^{\delta}\right]$.

In order to show that we have exactly S5 as the modal logic of grounds, we use Theorem 3 and observe that the switches of the proof of Theorem 6 still work.

We remark that if a universe has an independent family of upward switches and buttons, then so does any ground of that universe. This means that upward S4.2 is downwards necessary. Similarly, if a model has downward buttons and switches, then this remains upward necessary 9

Now, we consider the dual situation to that of Theorem 8 upward S5 and downward S4.2.

Theorem 9 If ZFC+ "there is an inaccessible cardinal $\delta$ in $\mathbf{L}$ such that $\mathbf{L}_{\delta}<\mathbf{L}$ " is consistent, then there is a model of set theory whose modal logic of forcing is S5 and whose modal logic of grounds is S4.2.

Proof sketch. Again, this proof is a combination of the constructions in Theorem 6 and [6, Theorem 5]. Start in $\mathbf{L}$ with $\mathbf{L}_{\delta}<\mathbf{L}$ and $\delta$ inaccessible in $\mathbf{L}$. Force to $\mathbf{L}[G]$ with the Easton support product $\mathbb{P}:=\prod_{\gamma \in \operatorname{Reg}} \mathbf{L} \operatorname{Add}(\gamma, 1)$, as in Theorem [6. As before in the proof of Theorem 8, we still have $\mathbf{L}_{\delta}\left[G_{\delta}\right]<\mathbf{L}[G]$ (as $\delta$ was inaccessible in $\mathbf{L}$ ). We can now appeal to the definability of forcing relation and the fact that $\mathbf{L}_{\delta}<\mathbf{L}$ : anything a condition $p$ forces over $\mathbf{L}$ with $\mathbb{P}$ is the same as what it forces over $\mathbf{L}_{\delta}$ with $\mathbb{P}_{\delta}$.

Finally, we perform the forcing to obtain upwards $\mathrm{S} 5$ from [6, Theorem 5] to obtain $\mathbf{L}[G][h]$. This forcing is the same as the Lévy collapse making $\delta$ into $\omega_{1}$. In $\mathbf{L}[G][h]$, we have upward S5. However, we also have downward .2: any ground of $\mathbf{L}[G][h]$ will contain some tail $\mathbf{L}\left[G^{\alpha}\right]$ just as in Theorem 6 , and thus we can take maximums as there to verify .2. But now we also have downward buttons and switches, just as in Theorem [ 6

Theorems 78 and 9 take care of three of the four possible distributions of the theories S4.2 and S5 to the upward and downward modal logics. This naturally raises the question whether it is possible to have S5 in both directions. We'll close this paper with the simple proof of the negative answer to this question:

${ }^{9}$ Unfortunately, we cannot conclude that downward S4.2 is upwards necessary, since we do not know whether downward .2 is valid in every model of set theory. 
Theorem 10 There is no model of set theory such that both its modal logic of forcing and its modal logic of grounds are S5.

Proof. Let us call an ordinal $\alpha$ a ground cardinal if there is a ground in which $\alpha$ is a cardinal. Let $\gamma$ be the least infinite ground cardinal; clearly, $\gamma \leqslant \omega_{1}$. The statement " $\gamma=\omega_{1}$ " is a downward button, but its negation is an upward button. So, if we had a model $V$ with $\operatorname{ML}(\Delta, V)=\mathrm{S} 5=\operatorname{ML}(\nabla, V)$, then $\gamma=\omega_{1}$ would have to be true by downward S5, but false by upward S5.

\section{References}

1. L. Esakia and B. Löwe. Fatal Heyting algebras and forcing persistent sentences. Studia Logica, 100(1-2):163-173, 2012.

2. S. Friedman, S. Fuchino, and H. Sakai. On the set-generic multiverse, 2012. submitted.

3. G. Fuchs. Closed maximality principles: implications, separations and combinations. Journal of Symbolic Logic, 73(1):276-308, 2008.

4. G. Fuchs. Combined maximality principles up to large cardinals. Journal of Symbolic Logic, 74(3):1015-1046, 2009.

5. G. Fuchs, J. D. Hamkins, and J. Reitz. Set-theoretic geology, 2011. submitted.

6. J. D. Hamkins. A simple maximality principle. Journal of Symbolic Logic, 68(2):527-550, 2003.

7. J. D. Hamkins. The Ground Axiom. Oberwolfach Reports, 2(4):3160-3162, 2005.

8. J. D. Hamkins. Some second order set theory. In R. Ramanujam and S. Sarukkai, editors, Logic and its applications. Third Indian Conference, ICLA 2009, Chennai, India, January 7-11, 2009. Proceedings, volume 5378 of Lecture Notes in Computer Science, pages 36-50. Springer, Berlin, 2009.

9. J. D. Hamkins. The set-theoretical multiverse. Review of Symbolic Logic, to appear.

10. J. D. Hamkins, G. Leibman, and B. Löwe. Structural connections between a forcing class and its modal logic, 2012. submitted.

11. J. D. Hamkins and B. Löwe. The modal logic of forcing. Transactions of the American Mathematical Society, 360(4):1793-1817, 2008.

12. J. D. Hamkins and W. H. Woodin. The necessary maximality principle for c.c.c. forcing is equiconsistent with a weakly compact cardinal. Mathematical Logic Quarterly, 51(5):493-498, 2005.

13. T. Jech. Set Theory. Springer Monographs in Mathematics. Springer-Verlag, Heidelberg, 3rd edition, 2003.

14. R. Laver. Certain very large cardinals are not created in small forcing extensions. Annals of Pure and Applied Logic, 149(1-3):1-6, 2007.

15. G. Leibman. Consistency strengths of modified maximality principles. $\mathrm{PhD}$ thesis, City University of New York, 2004.

16. G. Leibman. The consistency strength of $\operatorname{MP}_{\mathrm{CCC}}(\mathbb{R})$. Notre Dame Journal of Formal Logic, 51(2):181-193, 2010.

17. J. Reitz. The Ground Axiom. PhD thesis, City University of New York, September 2006.

18. J. Reitz. The Ground Axiom. Journal of Symbolic Logic, 72(4):1299-1317, 2007.

19. C. J. Rittberg. The modal logic of forcing. Master's thesis, Westfälische WilhelmsUniversität Münster, 2010. 
20. J. F. A. K. van Benthem. The logic of time. A model-theoretic investigation into the varieties of temporal ontology and temporal discourse, volume 156 of Synthese Library. D. Reidel Publishing Co., Dordrecht, 1983.

21. W. H. Woodin. The continuum hypothesis, the generic multiverse of sets, and the $\omega$ conjecture, 2009. preprint. 